\title{
How much can donor/acceptor-substitution change the responses of long push-pull systems to DC fields?
}

\author{
Michael Springborg ${ }^{a}$ and Bernard $\operatorname{Kirtman}^{b}$ \\ ${ }^{a}$ Physical and Theoretical Chemistry, \\ University of Saarland, 66123 Saarbrücken, Germany \\ ${ }^{b}$ Department of Chemistry and Biochemistry, \\ University of California, Santa Barbara, California 93106, U.S.A.
}

(Dated: October 25, 2018)

\begin{abstract}
Mathematical arguments are presented that give a unique answer to the question in the title. Subsequently, the mathematical analysis is extended using results of detailed model calculations that, in addition, throw further light on the consequences of the analysis. Finally, through a comparison with various recent studies, many of the latter are given a new interpretation.
\end{abstract}




\section{INTRODUCTION}

The response to external electric and magnetic fields provides a fundamental tool for studying and altering the properties of materials with numerous attendant applications. In particular higher-order responses allow for 'manipulating light with light.' Thus, there is considerable interest in identifying molecular systems with large non-linear responses.

One approach in this direction is based on push-pull systems, i.e., chain-like molecules with an electron-donor group at one end and an electron-acceptor group at the other (see Fig. 1). When the backbone is a $\pi$-conjugated oligomer the $\pi$ electrons of the backbone may respond easily to perturbations like those of the substituents and/or external fields. Due to the donor and acceptor groups a large electron transfer, and, accordingly, a large dipole moment can occur and one may hope for large responses of the dipole moment to external fields. For these $\pi$-conjugated systems, each circle in Fig. 1 could be, for example, a vinylene group, a phenylene group, a methinimine group, or combinations of those.

If the push-pull system is sufficiently large, we may split it into three parts, i.e., a left $(\mathrm{L})$, a central $(\mathrm{C})$, and a right $(\mathrm{R})$ part as shown in Fig. 1, Electrons of the central part are assumed to be so far from the terminations that they do not feel the latter (or, more precisely, the effects of the terminations are exponentially decaying in the central part).

The dipole moment, $\vec{\mu}$, is useful in quantifying the response of the system to an external electric field,

$$
\begin{aligned}
\mu_{i}(\omega)= & \mu_{i}^{(0)}(\omega)+\sum_{j} \sum_{\omega_{1}} \alpha_{i j}\left(\omega ; \pm \omega_{1}\right) \cdot E_{j}\left(\omega_{1}\right) \\
& +\frac{1}{2} \sum_{j k} \sum_{\omega_{1}, \omega_{2}} \beta_{i j k}\left(\omega ; \pm \omega_{1}, \pm \omega_{2}\right) \cdot E_{j}\left(\omega_{1}\right) E_{k}\left(\omega_{2}\right) \\
& +\frac{1}{6} \sum_{j k l} \sum_{\omega_{1}, \omega_{2}, \omega_{3}} \gamma_{i j k l}\left(\omega ; \pm \omega_{1}, \pm \omega_{2}, \pm \omega_{3}\right) \cdot E_{j}\left(\omega_{1}\right) E_{k}\left(\omega_{2}\right) E_{l}\left(\omega_{3}\right)+\cdots
\end{aligned}
$$

Here, $E_{m}\left(\omega_{s}\right)$ is the $m$ th component (i.e., $x, y$, or $z$ ) of the external field with the frequency $\omega_{s}$ and $\omega$ is the frequency of the response of the molecule to the field. The $\omega_{n}$ summations

go over all the frequencies of the applied field. $\mu_{i}^{(0)}(\omega)$ is the dipole moment in the absence of the field which vanishes for $\omega \neq 0$. Moreover, $\alpha_{i j}\left(\omega ; \pm \omega_{1}\right)$ is the linear polarizability, and $\beta_{i j k}\left(\omega ; \pm \omega_{1}, \pm \omega_{2}\right), \gamma_{i j k l}\left(\omega ; \pm \omega_{1}, \pm \omega_{2}, \pm \omega_{3}\right), \ldots$ are the first, second, .. hyperpolarizability. Sum rules require that these quantities can be non-zero only if the frequency of the response, $\omega$, equals the sum of the frequencies (eventually multiplied by -1 ), i.e., for 
$\gamma_{i j k l}\left(\omega ; \pm \omega_{1}, \pm \omega_{2}, \pm \omega_{3}\right)$ we require $\omega= \pm \omega_{1} \pm \omega_{2} \pm \omega_{3}$.

In the present paper we focus on static external fields, in which case $\omega_{i}=0$. Furthermore, we shall study a neutral system, although our arguments also are valid for charged systems as long as the extra charge is localized to the terminations. We let $\rho(\vec{r})$ be the (field-dependent) total charge density (i.e., the sum of the nuclear and electronic charge densities), and choose the long axis to be $z$. Then the component of the total dipole moment that is of interest here, namely $z$, is given by (omitting its argument, $\omega$ )

$$
\mu_{z}=\int \rho(\vec{r}) z d \vec{r}=\int_{L} \rho(\vec{r}) z d \vec{r}+\int_{C} \rho(\vec{r}) z d \vec{r}+\int_{R} \rho(\vec{r}) z d \vec{r},
$$

where we have split the integral into contributions from the left, central, and right regions of the chain. The central region consists of identical neutral units. We can, therefore, write

$$
\int_{C} \rho(\vec{r}) z d \vec{r}=K_{C} \mu_{C}
$$

where $K_{C}$ is the number of units in $\mathrm{C}$ and $\mu_{C}$ is the $z$ component of the dipole moment of one of these units. In order to evaluate the other two contributions to the total dipole moment in Eq. (2) we define a 'typical' center for each term, i.e., $\vec{R}_{R}$ and $\vec{R}_{L}$ (these could, e.g., be the center of mass of the right and left parts, respectively), and let $Z_{R}$ and $Z_{L}$ be the $z$ components of these vectors. Since the chain is neutral we, then, obtain

$$
\int_{L} \rho(\vec{r}) z d \vec{r}+\int_{R} \rho(\vec{r}) z d \vec{r}=\left(Z_{R}-Z_{L}\right) \int_{R} \rho(\vec{r}) d \vec{r}+\int_{L} \rho(\vec{r})\left(z-Z_{L}\right) d \vec{r}+\int_{R} \rho(\vec{r})\left(z-Z_{R}\right) d \vec{r} .
$$

The first term on the right hand side describes the contribution to the dipole moment associated with electron transfer from one end to the other. This term grows linearly with chain length (due to $Z_{R}-Z_{L}$ ) as does the term in Eq. (3). On the other hand, the last two terms in Eq. (4) describe local dipole moments that arise from the electron distributions within the two terminal regions and they are independent of the chain length.

This discussion suggests that donor/acceptor $(=\mathrm{D} / \mathrm{A})$ substitution at the ends of long chains may change the charge distribution in $\mathrm{R}$ and L so as to strongly enhance the dipole moment and, consequently, produce a particularly large change in the dipole moment when the system is exposed to an external electric field. Therefore, very many studies have been devoted to push-pull systems as a function of increasing length (see, e.g., [1, $, 2,3,4,5,5,6,7,8,9,9$, 10, 11, 12, 13, 14, 15, 16, 17, 18]).

Not only the electrons but also the structure (phonons) will respond to a static electric field. We will demonstrate that, for sufficiently long chains, the electronic response per unit 
of a push-pull system (with structural relaxation taken into account) becomes independent of the donor and acceptor groups, implying that the materials properties cannot be improved upon substitution. Our mathematical arguments for this finding are presented in the next section, and in Sec. III we illustrate and analyse the results through calculations on a model system. The particular case of inversion symmetry is discussed in Sec. IV] where we also make a comparison with previous results. Finally, a summary is provided in Sec. $\mathrm{V}$.

The arguments we present are related to those originally given by Vanderbilt and KingSmith for an extended system in the absence of an external field. They argued that the permanent polarization (i.e. dipole moment per unit length) is a bulk property $\underline{\underline{19}}$ Very recently, Kudin et al. .20 proved that the permanent polarization is quantized for $\mathrm{D} / \mathrm{A}$ substituted systems. Neither of these works considered the induced polarization or the structural relaxation due to an external field. Finally, in a recent paper we presented some of the arguments behind the present work but did not analyze the predictions as we do here using a model system. $\underline{21}^{2}$

\section{CHANGES IN THE CHARGE DISTRIBUTION UPON SUBSTITUTION}

By replacing some (groups of) atoms with others at the chain ends, the electronic orbitals with components near the ends will change. Since the set of electronic orbitals is orthonormal, all other orbitals will change as well. Accordingly, the charge distribution may change everywhere due to the substitutions.

When an electrostatic field is applied as well, each orbital will respond to the field. Since the orbitals will have changed due to the substitution, so will their responses to the field. Furthermore, the structural responses due to the field will also depend on the substitution at the ends. Therefore, the dipole moment can depend upon both the substitution and the field. From these arguments there is no reason to believe that $\mu^{(0)} / N, \alpha / N, \beta / N, \gamma / N$, ... (with $N$ being the number of repeated units) will be independent of the substitution. However, we shall argue here that the charge

$$
q=\int_{R} \rho(\vec{r}) d \vec{r}
$$

in Eq. (44) can change, at most, by an integral number of elementary units for different D/A substitutions at fixed external static field. Our proof is a generalization of arguments due 
to Vanderbilt and King-Smithำ (see also [20]), and was previously proposed by the present authors. ${ }^{21}$ It will be verified here by calculations on a model system and given a thorough analysis on that basis.

For a given system (with specified geometry), and value of the external field, we transform the set of occupied orbitals into a set of orthonormal, localized functions. Those functions ascribed to $\mathrm{C}$ will be similar to the Wannier functions of the infinite periodic system. The localized orbitals will be centered in one region, but may have tails sticking into another region. We assume that the terminal regions are large enough so that any functions centered therein, which differ from those of $\mathrm{C}$, are exponentially vanishing in $\mathrm{C}$. On the other hand, those functions ascribed to $\mathrm{C}$, but centered on units next to $\mathrm{L}$ or $\mathrm{R}$, will likely have tails extending into those regions.

The density matrix can then be written in block-diagonal form with three blocks, one for each of the three regions. Since the density matrix is idempotent, each block will be so, too, and there will be an integral number of electrons associated with each of the three sets of functions. That is to say, the number of electrons associated with the functions centered in the two end regions is integral. Accordingly, any non-integral part of $q$ is associated with the tails of the functions in $\mathrm{C}$ that extend into $\mathrm{R}$, which, per construction, is independent of the terminations, i.e., also of D/A substitution.

We conclude that, for different terminations, $q$ can change only by an integer. This is valid for long chains and all fields. Therefore, the electronic response per unit of the chains to the field, with or without nuclear response, is independent of termination. The only possible change for different terminations is that $q$ may jump by an integer for different field strengths. In fact, our numerical studies on a Hückel-type model will confirm this prediction.

Of course, in ab initio calculations, there may also be a jump due to changing the basis set or the method (e.g. Hartree-Fock vs. Kohn-Sham DFT).

\section{ILLUSTRATING AND ANALYZING THE RESULT}

In order to explore in detail the predictions from above we studied a Hückel like model for long, finite $(\mathrm{AB})_{2 K+1}$ chains. In our model, we use a basis set of orthonormal atomic orbitals (AOs) with one AO per atom. The system has one electron per atom, and the nuclei are given charges of +1 whereas the electronic charge is set equal to -1 . (All quantities 
are expressed in atomic units in this paper.) Given that $\chi_{n}$ is the AO of the $n$th atom $(n=1,2, \ldots, 4 K+2)$ and $\hat{h}$ is the Kohn-Sham or Fock single-electron hamiltonian we assume that only $\left\langle\chi_{j}|\hat{h}| \chi_{j}\right\rangle,\left\langle\chi_{j}|\hat{h}| \chi_{j \pm 1}\right\rangle$, and $\left\langle\chi_{j}|\hat{h}| \chi_{j \pm 2}\right\rangle$ are non-vanishing with values

$$
\begin{aligned}
\left\langle\chi_{2 p+1}|\hat{h}| \chi_{2 p+1}\right\rangle & =\epsilon_{0} \\
\left\langle\chi_{2 p}|\hat{h}| \chi_{2 p}\right\rangle & =-\epsilon_{0} \\
\left\langle\chi_{j}|\hat{h}| \chi_{j+1}\right\rangle & =-\left[t_{1}-\alpha_{1}\left(z_{j+1}-z_{j}\right)\right] \\
\left\langle\chi_{j}|\hat{h}| \chi_{j+2}\right\rangle & =-\left[t_{2}-\alpha_{2}\left(z_{j+2}-z_{j}\right)\right]
\end{aligned}
$$

Here $z_{j}$ is the position of the $j$ th atom. Different donor and acceptor groups are modeled by modifying the on-site energies of the terminating atoms and/or the terminating hopping integrals,

$$
\begin{aligned}
\left\langle\chi_{1}|\hat{h}| \chi_{1}\right\rangle & =\epsilon_{0}+\epsilon_{L} \\
\left\langle\chi_{4 K+2}|\hat{h}| \chi_{4 K+2}\right\rangle & =-\epsilon_{0}+\epsilon_{R} \\
\left\langle\chi_{1}|\hat{h}| \chi_{2}\right\rangle & =-\left[t_{1}-\alpha_{1}\left(z_{2}-z_{1}\right)\right]+t_{L} \\
\left\langle\chi_{4 K+1}|\hat{h}| \chi_{4 K+2}\right\rangle & =-\left[t_{1}-\alpha_{1}\left(z_{4 K+2}-z_{4 K+1}\right)\right]+t_{R} .
\end{aligned}
$$

Finally, we assume that

$$
\left\langle\chi_{j}|z| \chi_{k}\right\rangle=\delta_{j, k} z_{j}
$$

In order to analyse the results we, first, define a reference structure for which the position of the $n$th atom is

$$
z_{n}^{(0)}=\frac{a}{2}\left(n-2 K-\frac{3}{2}\right)-(-1)^{n} u_{0}
$$

Here $a$ is the length of the unit cell for an infinite, periodic system with the same electronic interactions and no external field. Subsequently, we define for each atom

$$
\begin{aligned}
u_{n} & =z_{n}-\frac{a}{2}\left(n-2 K-\frac{3}{2}\right) \\
\Delta z_{n} & =z_{n}-z_{n}^{(0)} .
\end{aligned}
$$

The total energy is written as the sum over occupied orbital energies (multiplied by 2 due to spin degeneracy) augmented by a harmonic term in the nearest- and the next-nearestneighbour bond lengths,

$$
E_{\mathrm{tot}}=2 \sum_{i=1}^{\mathrm{occ}} \epsilon_{i}+\frac{k_{1}}{2} \sum_{p=1}^{4 K+1}\left(z_{p+1}-z_{p}\right)^{2}+\frac{k_{2}}{2} \sum_{p=1}^{4 K}\left(z_{p+2}-z_{p}\right)^{2}-E_{\mathrm{DC}} \sum_{p=1}^{4 K+2} z_{p} .
$$


$E_{\mathrm{DC}}$ is the strength of the electrostatic field. For the infinite, periodic chain without an external field, the lowest total energy corresponds to a certain lattice constant $a$ and

$$
u_{n}=(-1)^{n+1} u_{0} .
$$

The force constants $k_{1}$ and $k_{2}$ are determined so that $a$ and $u_{0}$ take certain chosen values.

With

$$
\psi_{i}=\sum_{n=1}^{4 K+2} C_{n i} \chi_{n}
$$

being the $i$ th orbital (ordered according to increasing orbital energy) we calculate the Mulliken charge on the $n$th atom for field $E_{\mathrm{DC}}$ as

$$
q_{n}\left(E_{\mathrm{DC}}\right)=1-2 \sum_{i=1}^{2 K+1}\left|C_{n i}\right|^{2}
$$

which leads to the dipole moment

$$
\mu_{z}=\sum_{n=1}^{4 K+2} z_{n} q_{n}\left(E_{\mathrm{DC}}\right) .
$$

The charge transfer is given through

$$
q=\sum_{n=2 K+2}^{4 K+2} q_{n}\left(E_{\mathrm{DC}}\right) .
$$

We also define

$$
\begin{aligned}
& \Delta_{1} q_{n}\left(E_{\mathrm{DC}}\right)=q_{n}\left(E_{\mathrm{DC}}\right)-\tilde{q}_{n}(0) \\
& \Delta_{2} q_{n}\left(E_{\mathrm{DC}}\right)=q_{n}\left(E_{\mathrm{DC}}\right)-q_{n}(0) .
\end{aligned}
$$

where $\tilde{q}_{n}(0)$ is the charge for the infinite, periodic chain in the absence of the field. $\Delta_{2} q_{n}\left(E_{\mathrm{DC}}\right)$ quantifies the effects on the charge distribution of the push-pull chain due to including the field, whereas $\Delta_{1} q_{n}\left(E_{\mathrm{DC}}\right)$ includes effects both from the field and from the terminations. Note that $\Delta_{1} q_{n}\left(E_{\mathrm{DC}}\right)-\Delta_{2} q_{n}\left(E_{\mathrm{DC}}\right)$ gives the field-independent effect of the terminations. Finally, it turns out to be useful to define the center and width of the $i$ th orbital according to

$$
\begin{aligned}
\zeta_{i} & =\sum_{n=1}^{4 K+2} z_{n}\left|C_{n i}\right|^{2} \\
\Delta \zeta_{i} & =\left[\sum_{n=1}^{4 K+2}\left(z_{n}-\zeta_{i}\right)^{2}\left|C_{n i}\right|^{2}\right]^{1 / 2}
\end{aligned}
$$


which is consistent with Eq. (8) .

We performed calculations for six different terminations specified by $\left(\epsilon_{L}, \epsilon_{R}, t_{L}, t_{R}\right)$. The results are summarized in Figs. 2, 3, 4, 5, and 6. Since our model is that of a finite chain with two different types of atoms, $\mathrm{A}$ and $\mathrm{B}$, the Mulliken charges in the central region take two values. This is clearly recognized in the presentation of $q_{n}$ in Fig. 2 for $E_{\mathrm{DC}}=-0.015$. In Fig. 2 it is also seen that near the ends, the Mulliken charges differ from the values of the inner part and, moreover, these charges depend sensitively on the terminations. For the field strength $E_{\mathrm{DC}}=-0.015$ these findings are only marginally modified compared to those of a vanishing field (not shown). From $\Delta_{1} q_{n}$ we see that the combination of electrostatic field and termination leads to an internal polarization of each unit in C. Actually, $\Delta_{1} q_{n}$ shows a reduced internal polarization compared to $\Delta_{2} q_{n}$. Thus, terminating the chain reduces the effect of the field in that regard. Whereas $\Delta_{2} q_{n}$ contains information about the field-induced charge redistributions, $\Delta_{1} q_{n}$ contains additional information about the (field-dependent) effects of the terminations. For $E_{\mathrm{DC}}=-0.015$ the field-induced charge redistributions are smaller near the terminations than in the central parts.

For the larger field, $E_{\mathrm{DC}}=-0.03$, in Fig. 3 the identification of the central region becomes much more difficult and, as we shall see below, electrons are transferred from one end to the other. Moreover, in this case the field perturbs the system so strongly that the effects of the field are stronger than those of the terminations. This can be seen from the fact that $\Delta_{1} q_{n}$ and $\Delta_{2} q_{n}$ are very similar.

The structure also depends upon the termination. For the intermediate field of Fig. 2 (and for zero field as well) the atomic coordinate $u_{n}$ is nearly constant in $\mathrm{C}$ but varies considerably near the ends where its value depends on the termination, as was the case for the atomic charges. For the higher field in Fig. 3 it appears as if no central region can be identified from this parameter. However, the fact that $\Delta z_{n}$ is essentially linear for the innermost atoms implies that there is a well-defined, repeating structure in $\mathrm{C}$ with a lattice constant differing from that of the field-free case.

Fig. 4 shows that the charge transfer, $q$, is independent of termination (though not independent of the field), with the exception of jumps by (even) integers. (The integers are even because we have not allowed for spin polarization.) However, the charge distribution inside $\mathrm{R}$ or $\mathrm{L}$ does depend on the terminations and, as a consequence, the dipole moment does as well. On the other hand, the variation of $\mu_{z} / N$ as a function of $E_{\mathrm{DC}}$ for different termina- 
tions follows parallel curves, implying that the (hyper)polarizabilities are independent of the terminations. In fact, a least squares fit yields the values (including maximum deviations): $\mu_{0} / N=0.3245 \pm 0.0023, \alpha / N=1.677 \pm 0.013, \beta / N=18.17 \pm 0.20$, and $\gamma / N=606.9 \pm 9.1$ for all six terminations.

As a function of field $\mu_{z}$ is discontinuous and the power series expansion is valid only up to the field where the discontinuity occurs. Once such a discontinuity has been passed, the dipole moment depends more strongly on the field. This means that the only way of increasing the responses of long push-pull systems to DC fields is to design chains for which the integral electron transfers occur at low fields.

At a given field the size of the chain for which jumps in the charge $q$ (i.e. Zener tunneling) take place depends on the terminations (cf. Fig. (5)). In the shortest chains, for which Zener tunneling does not occur, $\mu_{z}$ follows parallel curves as a function of chain length, $N=2 K+1$, for different terminations. This means that the dipole moment and (hyper)polarizabilities per unit become independent of termination. However, as seen in Fig. 15, the slope of these curves increase after Zener tunneling has taken place, implying that the dipole moment increases. Assuming that the field-dependence of the dipole moment likewise increases, this suggests that the polarizability and/or hyperpolarizabilities per unit may increase for D/A substituted systems after an integral number of electrons has been transferred from one end to the other.

In Fig. 6 we show an example of what happens to the molecular orbitals when the jumps take place. Calculations were performed for field strengths between -0.0340 and -0.0485 in steps of -0.0005 , but in the figure we only show the results for fields where Zener tunneling occurs. In all cases, the curves vary smoothly as a function of field strength. At the lowest two fields, the occupied orbitals closest to the Fermi level have a center in the left part $\left(\zeta_{i}<0\right)$, whereas the unoccupied orbitals closest to the Fermi level are centered in the right part. At the field $E_{\mathrm{DC}} \simeq-0.0375$, two electrons (one per spin direction) are transferred from one side to the other, which again happens at a larger field $\left(E_{\mathrm{DC}} \simeq-0.0475\right)$. In the first case, we observe the occurrence of two new, very localized, orbitals close to (but not at) the Fermi level. The energetically lower (i.e occupied) one is localized towards to the chain end on the right side while the other (unoccupied) is localized towards the chain end on the left side. Accompanying this interchange is a similar interchange of two rather delocalized orbitals, both of which are further away from the Fermi level and centered closer to the 
middle of the chain. Again, at the second electron transfer a pair of new, rather localized, orbitals near (even closer to) the Fermi level show up towards the chain ends, and also this transfer is accompanied by some reorganization of the other orbitals. Finally, Fig. 6] also shows an example of a reorganization of the orbitals, i.e., for a field around $E_{\mathrm{DC}}=-0.0430$. Here, one localized, occupied orbital interchanges order with an adjacent (in energy) more delocalized orbital, but otherwise no further significant changes are observed.

\section{INVERSION SYMMETRY AND COMPARISON WITH PREVIOUS RE- SULTS}

Before proceeding to compare with previous results we develop an interesting consequence of our findings with regard to inversion symmetry. The same arguments can be applied for a system containing a mirror plane perpendicular to the chain axis, but here we shall for the sake of simplicity restrict ourselves to the case of inversion symmetry. Suppose the long oligomer of interest contains a central region made up of units with inversion symmetry. Even if the central part does not have inversion symmetry, it may be possible to create such with the addition of appropriate terminating groups. This is, for example, the case for oligomers of thienyleneethynylenes and thienylenevinylenes that were studied by Geisler et $a l . \underline{6}$ Many of the systems of interest fall into one of these two categories. Since, according to our findings, D/A substitution cannot affect the (hyper)polarizabilities per unit, the latter must vanish even if the symmetry is not preserved. For instance, modifying the terminations of the systems of Geisler et al. so that inversion symmetry no longer exists cannot result in a non-vanishing $\beta / N$ if the chains are sufficiently long.

A large fraction of previous observations are for systems of the type described in the preceding paragraph. Some of these cases are discussed below along with others pertinent to our findings herein. We now briefly consider, in particular, the works mentioned in the Introduction.

In their combined experimental and theoretical study on some push-pull oligoenes, Mey-

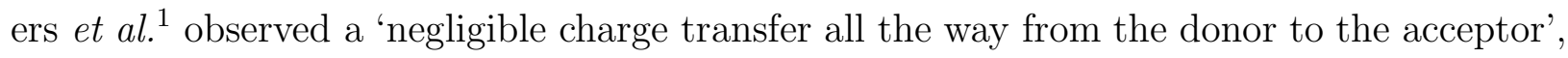
which implies that $q$ is independent of the termination. On the other hand, in their theoretical study Tsunekawa and Yamaguchi ${ }^{2}$ examined shorter, nitrogen-containing push-pull oligomers. They noted that these systems are interesting from the perspective of maximizing 
$\beta$, but our results establish that, for such to be true, the systems must be short enough so that our approach is inapplicable. This serves to highlight the point that apparent, but not real, discrepancies can occur due to shortness of the chain length.

Marder et al. $\frac{3}{.}$ presented an approach for unifying the description of linear and nonlinear polarization in organic polymethine dyes. It has since been shown that their analysis is invalid if phonons are taken into account $\frac{16}{}$ Here, however, we emphasize that the conclusions they draw regarding $\beta$ can, again, hold only for systems that are too short for our treatment to apply.

Clearly, the chain length required for validity of the treatment given here is an important issue. In Fig. [5 the dipole moment is converged for chains with some 20 units. However, this may be an artifact of our simple Hückel model. In an experimental study $\underline{\underline{4}}^{\underline{4}}$ and in several computational studies, $\underline{5}, \underline{9}, 10,14,22$ the second hyperpolarizability per unit was found to converge considerably slower which, in fact, agrees with our own earlier findings. .11 Thus, when focusing on higher-order non-linear responses quite large chains may be required for the results of the present work to be relevant. In shorter push-pull systems (for instance those considered by Geisler et al. $\underline{6,11}$ or by Morley et al. $\underline{\underline{7.8}}$ ) D/A substitution can have an influence on the response.

As shown numerically by Champagne et al.,$\underline{12} \beta / N$ also converges relatively slowly as a function of size. They considered D/A substituted oligomers of polymetheimine [also called polycarbonitrile, $\left.(\mathrm{CHN})_{x}\right]$. This system has a zigzag backbone of alternating $\mathrm{C}$ and $\mathrm{N}$ atoms with alternating bond lengths. Without the bond length alternation it would, at least hypothetically, be possible to choose donor and acceptor groups so that the overall system is centrosymmetric. Even if chemical arguments imply that this structure is unrealistic, a non-zero value of $\beta / N$ for long chains should be ascribed, strictly speaking, to the bond length alternation.

Polyphenylenes and polypyridines have been studied by Zhang and Lu. $\underline{\underline{13}}$ They focused on $\alpha$ and $\gamma$ as a function of the length of a closed ring for each system and applied a finitefield approach in their calculations. Unfortunately, as we have shown earlier (see, e.g., [21]), this approach will never converge to the results for the infinite, periodic chain. Nevertheless, although $\beta / N$ will vanish for the polyphenylenes, we predict that a non-zero value will occur for both short and long oligomers of the polypyridines.

For the D/A substituted polyenes studied by Champagne et al $\underline{\underline{15}}$ our analysis confirms 
their findings, i.e., that $\beta / N$ will vanish for sufficiently large chains. Their numerical results indicate that $\beta / N$ goes through a maximum and that convergence to the infinite chain result for larger $N$ is slow.

Even the polarizability, $\alpha / N$, and the permanent dipole moment, $\mu_{z}^{(0)} / N$, may converge more slowly, as a function of chain length, than predicted by our simple model. This is, for example, the case for the systems investigated by Smith et al. $\stackrel{18}{ }$ and by Kudin et al. ${ }^{20}$

In a recent study, Botek et al. $!^{17}$ compared finite oligomers of $[N]$ helicenes and $[N]$ phenylenes that possess a helical structure for $N$ larger than roughly 6 . By making explicit use of the helical symmetry of the central region we predict that, when those systems are sufficiently long, D/A substitution will not be able to modify the electronic responses to static fields. The fact that Botek et al. find changes upon D/A substitution implies that the chains of their study are not converged to the long chain limit.

\section{SUMMARY}

As long as the applied field is not so strong that an integral number of electrons is transferred from one end to the other, the answer to the question of the title is clearly: there can be no change. This comes from our mathematical analysis in Sec. II, which generalizes treatments presented previously by Vanderbilt and King-Smith $\frac{19}{}$ and by Kudin et al. ${ }^{20}$ who considered only electronic polarization in the absence of an external electrostatic field. It is also in agreement with our own earlier prediction. ${ }^{21}$

Calculations on a model system confirm the basic result and shed light on the nature of the end-to-end charge transfer. Although the end charges, permanent dipole moment, and structure depend sensitively on the terminations neither the amount of charge transferred nor the (hyper)polarizabilities per unit do so. The field and/or chain length at which the charge jumps take place also depend on the terminations. Each jump is associated with an interchange of occupied and unoccupied molecular orbitals that are well-localized in the chain end region. These orbitals are close to but not at the Fermi level. There is also an accompanying orbital reorganization.

One consequence of our finding is that long unsubstituted chains which have inversion or mirror symmetry, or can be made symmetric by substitution, must have a vanishing first hyperpolarizability per unit. Experimental and theoretical determinations are consistent 
with this fact, although apparent contradictions can occur for short chains.

\section{Acknowledgments}

This work was supported by the German Research Council (DFG) through project Sp439/20 within the SPP 1145. Moreover, one of the authors (MS) is very grateful to the International Center for Materials Research, University of California, Santa Barbara, for generous hospitality.

1 F. Meyers, J. L. Brédas, and J. Zyss, J. Am. Chem. Soc. 114 (1992) 2914.

2 T. Tsunekawa and K. Yamaguchi, J. Phys. Chem. 96 (1992) 10268.

3 S. R. Marder, C. B. Gorman, F. Meyers, J. W. Perry, G. Bourhill, J.-L. Brédas, and B. M. Pierce, Science 265 (1994) 632.

4 I. D. W. Samuel, I. Ledoux, C. Dhenaut, J. Zyss, H. H. Fox, R. R. Schrock, and R. J. Silbey, Science 265 (1994) 1070.

5 S. Mukamel, A. Takahashi, H. X. Wang, and G. Chen, Science 266 (1994) 250.

6 T. Geisler, J. C. Petersen, T. Bjørnholm, E. Fischer, J. Larsen, C. Dehu, J.-L. Brédas, G. V. Tormos, P. N. Nugara, M. P. Cava, and R. M. Metzger, J. Phys. Chem. 98 (1994) 10102.

7 M. G. Hutchings, I. Ferguson, D. J. McGeein, J. O. Morley, J. Zyss, and I. Ledoux, J. Chem. Soc. Perkin Trans. 2 (1995) 171.

8 J. O. Morley, J. Chem. Soc. Perkin Trans. 2 (1995) 177.

9 Y. Verbandt, H. Thienpont, I. Veretennicoff, and P. Geerlings, Chem. Phys. Lett. 251 (1996) 47.

10 D. Lu, B. Marten, M. Ringnalda, R. A. Friesner, and W. A. Goddard III, Chem. Phys. Lett. 257 (1996) 224.

11 T. Bjørnholm, D. R. Greve, T. Geisler, J. C. Petersen, M. Jayaraman, and R. D. McCullough, Adv. Mat. 8 (1996) 920.

12 B. Champagne, D. Jacquemin, J.-M. André, and B. Kirtman, J. Phys. Chem. A 101 (1997) 3158.

13 Y. Zhang and Z.-H. Lu, J. Mol. Struct. (Theochem) 467 (1999) 233. 
14 M. Schulz, S. Tretiak, V. Chernyak, and S. Mukamel, J. Am. Chem. Soc. 122 (2000) 452.

15 B. Champagne, E. A. Perpète, D. Jacquemin, S. J. A. van Gisbergen, E.-J. Baerends, C. SoubraGhaoui, K. A. Robins, and B. Kirtman, J. Phys. Chem. A 104 (2000) 4755.

16 B. Kirtman, B. Champagne, and D. M. Bishop, J. Am. Chem. Soc. 122 (2000) 8007.

17 E. Botek, B. Champagne, M. Turki, and J.-M. André, J. Chem. Phys. 120 (2004) 2042.

18 S. M. Smith, A. N. Markevitch, D. A. Romanov, X. Li, R. J. Levis, and H. B. Schlegel, J. Phys. Chem. A 108 (2004) 11063.

19 D. Vanderbilt and R. D. King-Smith, Phys. Rev. B 48 (1993) 4442.

20 K. N. Kudin, R. Car, and R. Resta, J. Chem. Phys. 127 (2007) 194902.

21 M. Springborg and B. Kirtman, Phys. Rev. B (in press).

22 B. Kirtman, J.L. Toto, K.A. Robins and M. Hasan, J. Chem. Phys. 102 (1995) 5350. 

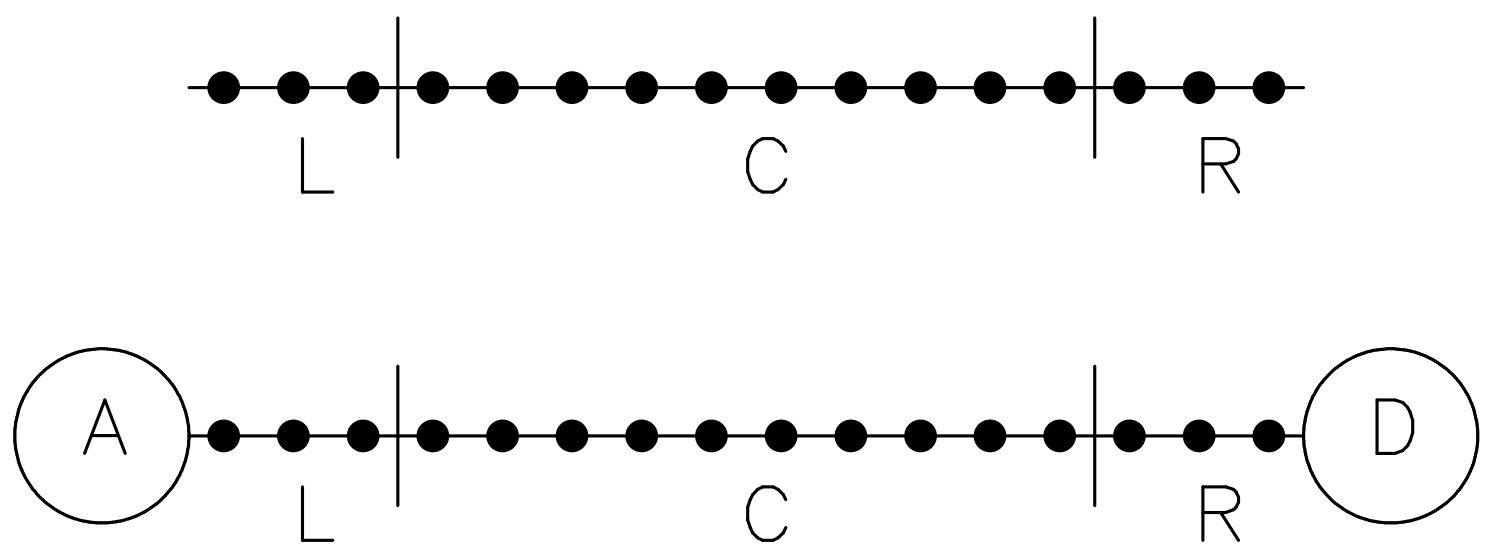

FIG. 1: Schematic representation of a long finite chain separated into a central region and two terminal regions. The lower chain shows what happens when a donor and an acceptor group have been added at the terminations. Each dot represents a group of atoms. 


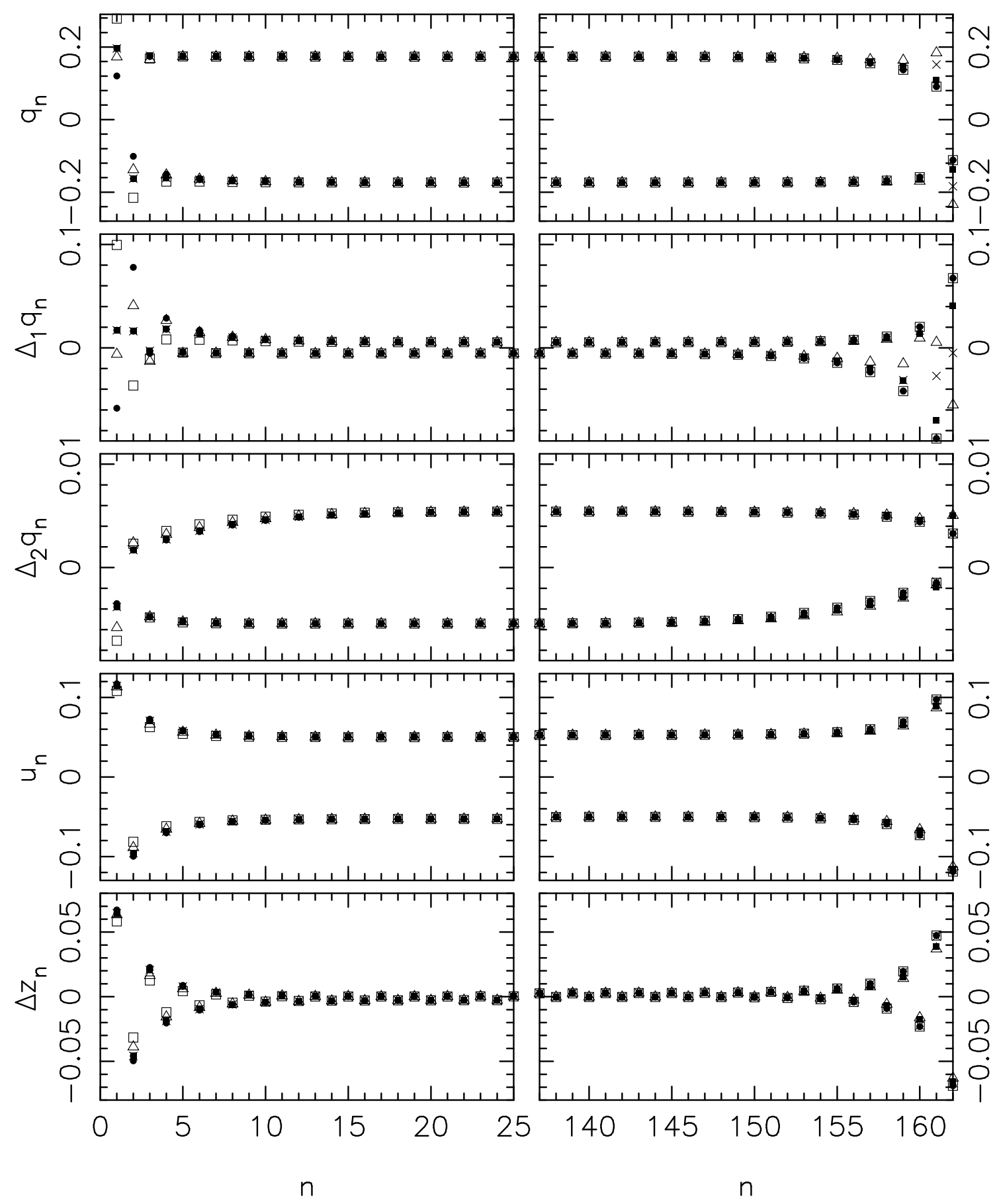

FIG. 2: Results from the model calculations for chains with $K=40$, i.e., 162 atoms and an external field of -0.015 a.u. The first three rows show quantities related to the charge distribution, i.e., those of Eqs. (14) and (17), whereas the two lowest rows show quantities related to the structure, i.e., those of Eq. (10). In all cases, $\epsilon_{0}=0.5, t_{1}=2.5, \alpha_{1}=4.5, t_{2}=0.5$, and $\alpha_{2}=0.2$. Moreover, $k_{1}$ and $k_{2}$ were chosen so that the optimized structure for vanishing field and an infinite periodic chain has $a=2.1$ and $u_{0}=0.05$. Open circles, closed circles, crosses, open squares, closed squares, and open triangles show results for which $\left(\epsilon_{L}, \epsilon_{R}, t_{L}, t_{R}\right)$ has been set equal to $(0,0,0,0),(0,0.5,0,0)$, $(0.5,0,0,0),(0,0,0,0.8),(0,0,0.8,0)$, and $(0.5,0.5,0.8,0.8)$, respectively. 


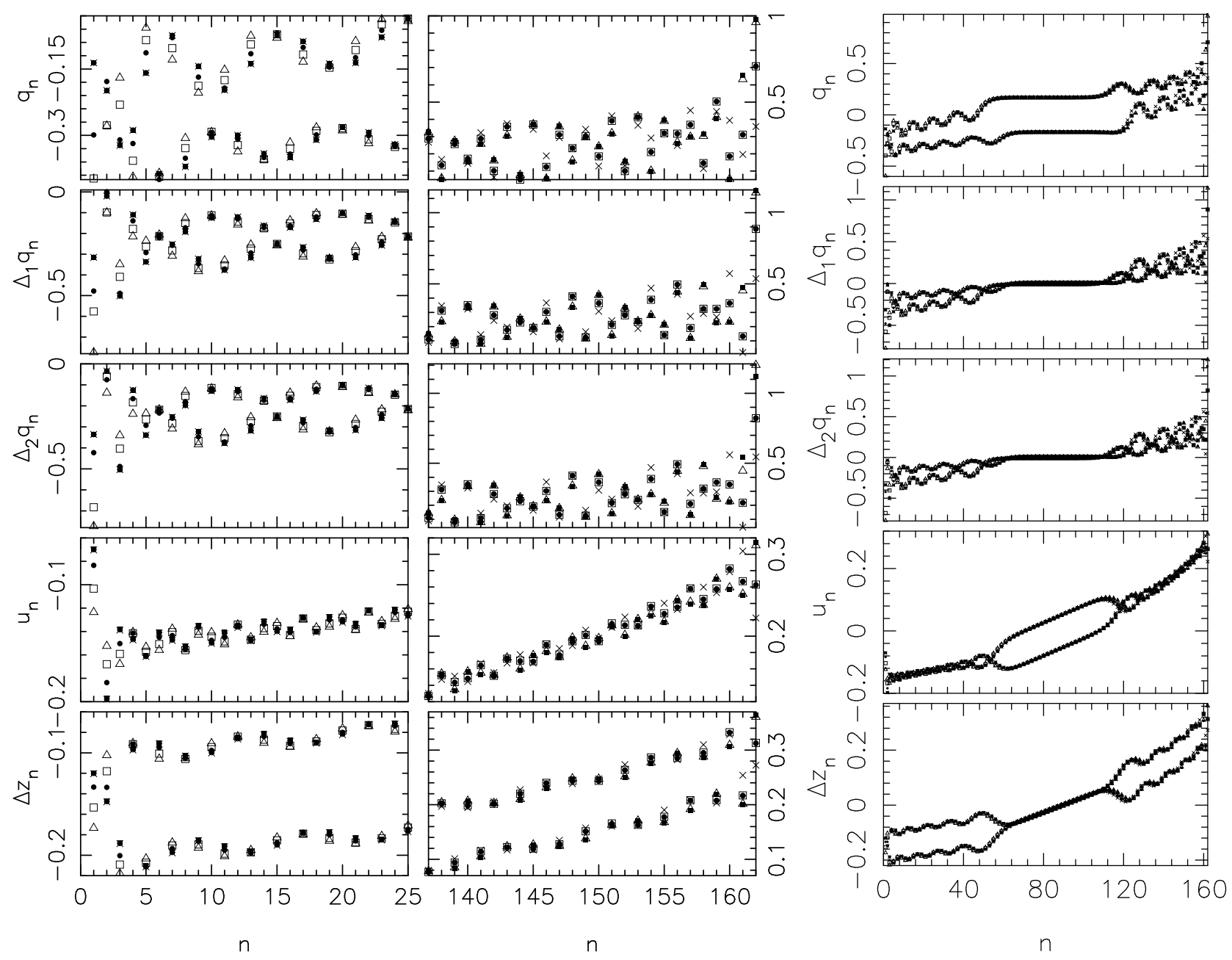

FIG. 3: Results from the model calculations for chains with $K=40$, i.e., 162 atoms and an external field of -0.03 a.u. The left and middle columns show results for the two ends whereas the right column shows the results for the whole chain. The presentation is as in Fig. 2 . 


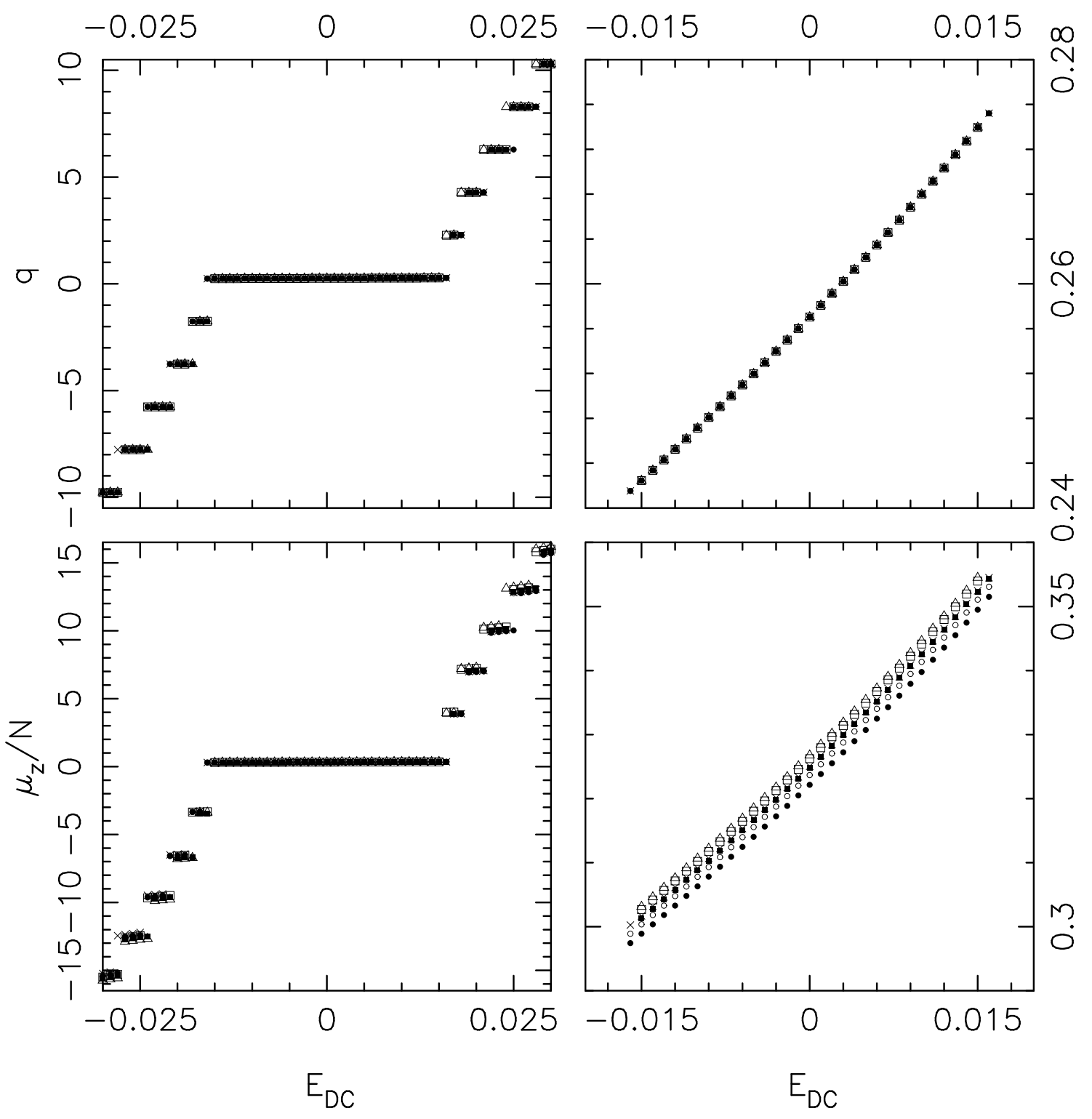

FIG. 4: The charge transfer, $q$, and the dipole moment per unit, $\mu / N$ with $N=2 K+1$, for the same systems as in Fig. 2, The right panels show a magnification of the left ones. 


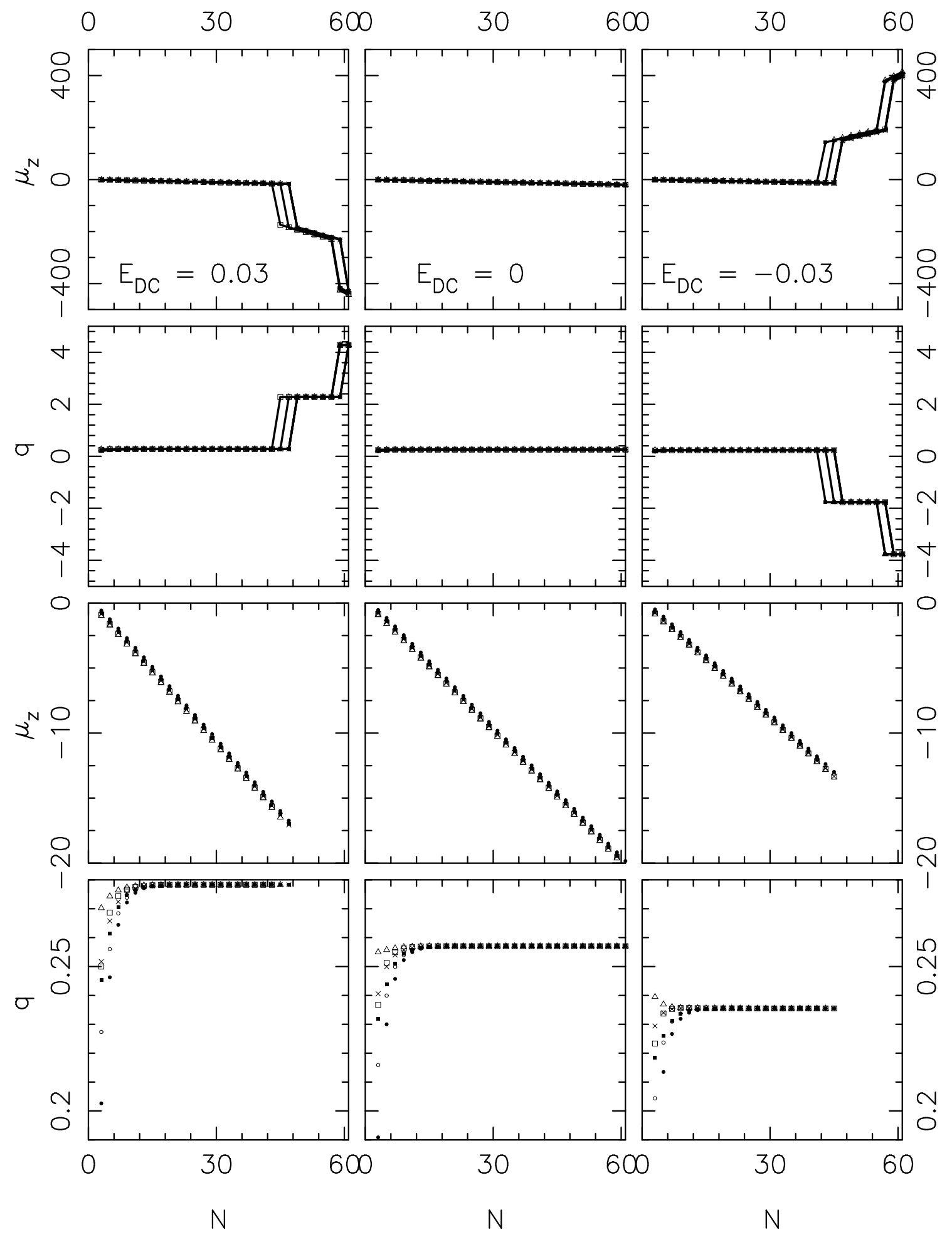

FIG. 5: The charge transfer, $q$, and the dipole moment $\mu$ with $N=2 K+1$, for the same systems as in Fig. 2 as functions of $N$. The two lower rows show a magnification of the two upper ones. The field strength has been set equal to $0.03,0$, and -0.03 for the left, middle, and right columns, respectively. 

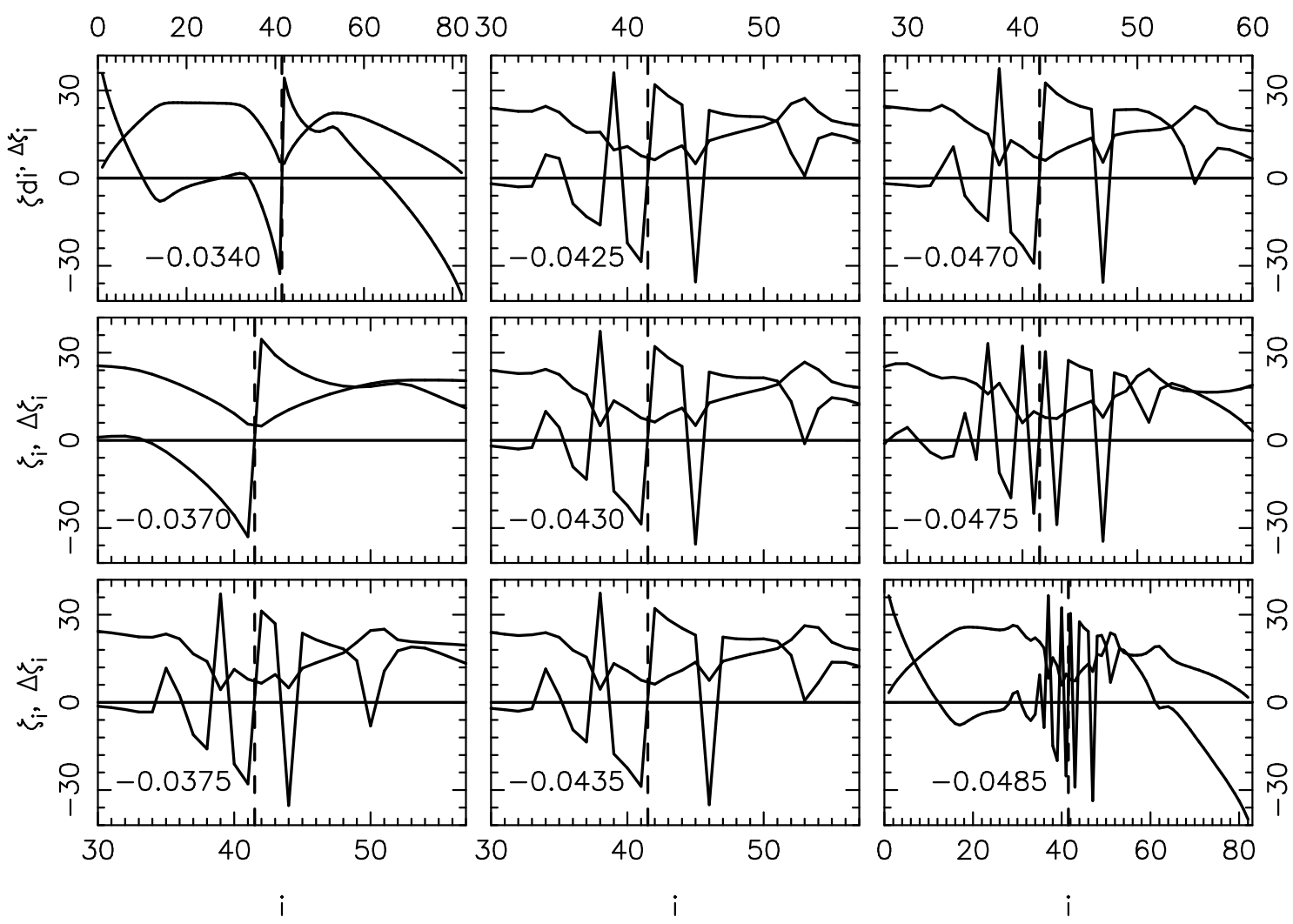

FIG. 6: $\zeta_{i}$ and $\Delta \zeta_{i}$ as a function of orbital index for a given push-pull chain with different fields. The vertical dashed lines separate occupied and unoccupied orbitals; $K=20$; and the parameter values are the same as in Fig. 2 except that only the first termination is considered. The field strength is given in each panel. Since $\Delta \zeta_{i} \geq 0$ the two curves are readily distinguished. Moreover, the panels for $E_{\mathrm{DC}}=-0.0340$ and -0.0485 have the same orbital index scales, as is the case for the panels for $E_{\mathrm{DC}}=-0.0370,-0.0375,-0.0425,-0.0430$, and -0.0435 , and for the panels for $E_{\mathrm{DC}}=-0.0470$ and -0.0475 . 